\title{
Homeopathy and its scope in increasing sperm count and its action on male fertility
}

Volume 6 Issue 6 - 2017

Keywords: asthenoteratozoospermia, asthenospermia, asthenozoospermia, ligoasthenoteratozoospermia, oligozoospermia, oligospermiea, polyzoospermia, teratospermia

\section{Opinion}

Hormonal imbalance, abnormal sperm shape, vericocele and Teratozoospermia are terms associated with male fertility. Low sperm count-Oligospermia- is a condition where less than 20million sperm/ $\mathrm{ml}$ are present in the ejaculate.

Nill sperm count/Azoospermia it is the term given to condition when there is absence of sperm/spermatozoa in the ejaculation.

\section{Low motility-oligospermaesthenia}

Oligospermia - Low Sperm Count - Oligospermia defined as less number of sperm in the ejaculate of the male or less than 20million sperm per millilitre. Normal Sperm count: $20 \mathrm{million} / \mathrm{millilitre}$ to 120 million /milliliter. Sperm count below 20 million $/ \mathrm{ml}$ called Oligospermia. Azoospermia is defined as the absence of spermatozoa in the ejaculation.

Homeopathic medicine is very effective in the treatment of Low Sperm Motility, Low Sperm Count, Low Sperm Volume, Semen Viscosity, Abnormal Sperm cell Morphology, Anti Sperm Antibodies and combination of above ailments. As per Clinical data analysis done by Dr. Abhishek M.D (Homeopathy) infertility in males and Low sperm count is very common then most couples think. In India, the mindset is so developed, that in case a female is not getting pregnant, we tend to think it's may be due to infertility on the part of female. But as per research, chances of male factor infertility are relevantly more. As per Dr. Abhishek M.D-Homeopathy, if any couple is trying to conceive from 1year, and not getting results positive for pregnancy, then both male and female should get them tested for infertility. We will be discussing in detail regarding male infertility here, Sperm count of 20million or more along with healthy motility and morphology of the sperm is termed as normal.

If a male find his sperm levels is less than 20million or you are having issues with sperm morphology, motility or mobility then Homeopathy medicines can helpful to enhance sperm quality and quantity in most natural way.

\section{Main causes of low sperm count}

a. Electromagnetic frequencies: Research studies have proven that EMF, leads to rise in testicles temperature, which there by decrease sperm count. Dr. Abhishek advice not to keep mobile phones in the pocket.

b. Radio frequency electromagnetic waves: Clinical study shows that RF-EMWs emitted from wi-fi connectivity device damage sperm and plays a important role in decreasing sperm production and motility. RF-EMWs lead to increasing sperm DNA fragmentation.

\author{
Abhishek Kasana \\ Department of Homeopathy, India \\ Correspondence: Abhishek Kasana, B.H.M.S, C.F.N-Delhi, D.I \\ Hom -United Kingdom, P.G.D.P.H- Chennai, M.D Homeopathy- \\ Maharashtra, India, Email drabhishek.kasana@gmail.com
}

Received: March 25, 2017 | Published: May 18, 2017

c. Tobacco smoking: Tobacco smoking habit damages the morphology of sperm. But the good part is that harm done by tobacco smoking can be reversed, once a person quit smoking.

d. Pesticides: Now a day there is huge exposure to insecticides and pesticides in our food. Research study shows that pesticides mimic estrogens hormone and alter health production of sperms.

e. Meat and dairy products: Dairy animals are highly exposed to high level of estrogen hormone to increase the productivity, hence milk, milk products or animal meat of such animal alter normal semen production.

f. Soy foods: Isoflavones, is a type of phytoestrogen which is commonly found in processed soy food, and research study have shown that isoflavone block estrogen receptor sites, which are required for testosterone

g. Alcohol: Clinical study at Aura Homeopathy clinic shows that alcohol consumption for long term leads to decrease in quality and quantity of sperms. Hence alcohol consumption should be stopped completely.

h. Plastics: Avoide use of plastic utinsils and container, as per studywhen plastic utensils are over heated it release xenohormones and xenohormones mimic estrogen hormone in human body.

i. Hyperthermia: Dr. Abhishek advice that overheats may damage sperm, hence frequent use of hot tub bath and saunas would be avoided. Further tight undergarments must be strictly avoided. Testicles hang to regulate the temperature which is favourable for healthy sperm production, and no-a day's males wear tight clothing, even during sleep.

j. Stress: Mental Stress leads to hormone imbalance, which affects production of sperm.

\section{Few tips given by dr. abhishek}

i. Get your sperm analysis test every 3rdmonth.

ii. Avoide exposure to Electromagnetic frequencies, Radio frequency electromagnetic waves, insecticides, pesticides, tobacco smoking, food rich in soy, caned or packed food, alcohol, 
plastics utensils and cary bags, tight undergarments, hot tub bath, long duration bike riding and reduce mental stress.

iii. Eat healthy diet rich in nutrients good for sperm production and male reproductive system. These are selenium, zinc, vitamin B12, folic acid, L-Carnitine, vitamin $\mathrm{E}$ and food rich in antioxidants.

iv. Get your male Hormone in Balance-Men can also get affected by hormonal imbalance. Testosterone is one of the main male hormones, and due to present modern lifestyle it become imbalanced.

Few pollutants which "mimic" estrogens for example xenohormones in human body results in decreasing testosterone. Further increased estrogen level in males may lead to Decreased libido or sex drive, ED- Erectile dysfunction, Decrease or low sperm count, and Decrease seminal fluid production.

\section{Few tips for balancing male hormones}

i. Avoid your exposure to xenohormones.

ii. Avoid non-veg food or Opt. for organic meats and dairy.

iii. Avoid eating soy foods.

iv. Detoxify your body with lots and lots of water and juices.

v. Increase intake of fiber and cruciferous vegetables. Crociferous vegetables contain certain element that helps the body to clean itself of estrogen excess.

vi. Avoide Influx of xenohormones: By avoiding foods containing soy protein, by avoiding Pesticides in food, by avoiding Estrogen hormones given to dairy animals and lastly by avoiding Use of plastic utensils

\section{Homeopathy for male fertility \& good sperm quality and quantity}

Following natural homeopathic medicine have shown there curable effects on oligospermia and morphological defects of sperms. Studies have shown that homeopathy medicine helps male having healthier sperm with increase chances of fertility.

These homeopathic medicines are adapt gens which help normal functioning of endocrine system. For best results use homeopathic medicines at least for 6 months, and do cross check your sperm count and analysis every 3 months.

\section{Advantage of homeopathy treatment}

i. Homeopathy is very fast to act and one of the natural and safest modes of treatment.

ii. Proven and tested in thousands of infertility patients with success rate of $85 \%$.

iii. Homeopathic Medicine not only effective in increasing sperm count but also helps in increasing sperm motility and sperm Volume, abnormal sperm morphology can also be corrected with homeopathy treatment.

iv. Homeopathic medicine does not contain any hormones.

v. Minimum time required for homeopathy treatment is $3-4$ month with at least 1 million sperm count, further there is no need of medicine for at least 7 to 8year after completion of homeopathy treatment.

vi. Homeopathy also treats Varicocele, imbalance of hormones and other related urological abnormalities. vii. Homeopathy promotes Spermatogenesis.

viii. It not only helps to achieve normal sperm counts in males with Small Testes but also in those male having only one testis.

\section{Frequently asked questions}

\section{A. What is homeopathy fertility treatment?}

Homeopathy helps in Conceiving naturally, at aura we provide researched based homeopathic medicine based on exhaustive research. Medicine is selected on the basis of individualization, which include totality of symptoms- Considering both mental and physical symptoms. As per Dr. Abhishek it is tailored or personalized treatment. It is natural, very safe, and gentle without any side-effects.

Homeopathy treatment for infertility, is best way of conceiving naturally. Along with male infertility it also take cares of:

a. PCOS.

b. Endometriosis.

c. Fallopian Tubes blockage.

d. Unexplained Infertility.

e. Hormonal Imbalances and problem related to Ovulation

f. Uterine Fibroids and Cysts.

g. Secondary Infertility.

h. History of Multiple Repeated Miscarriages.

\section{B. How long will it take for me to conceive naturally?}

Duration of treatment depend from person to person. Minimum time required for homeopathy treatment is $3-4$ month with at least 1 million sperm count. Treatment provides individualized homeopathic therapy for male infertility. The selected homeopathic medicine is prescribed on the basis of symptoms totality. With course of treatment sperm quality and quantity improves significantly.

\section{What are the side-effects of homeopathy fertility treatment} protocol?

No side effect noted.

\section{Can it help in increasing my sperm count?}

Yes, homeopathy treatment is very capable of increasing sperm count, further Dr. Abhishek advice each and every patient to cross check sperm count every 3rdmonth so as to monitor the success of the treatment.

\section{Best homeopathic medicines for oligospermia}

Many homeopathic remedies have been known to cure Low sperm Count. Some of these are;

a. Agnus castus: Sexual melancholy. Fear of death. Sadness with impression of speedy death. Absentminded, forgetful, lack of courage. Illusion of smell-herrings, musk. Nervous depression and mental forebodings. Yellow discharge from urethra. No erections. Impotence. Parts cold, relaxed. Desire gone (Selen; Con; Sabal). Scanty emission without ejaculation. Loss of prostatic fluid on straining. Gleety discharge. Testicles, cold, swollen, hard, and painful.

b. Anacardium orientalis: Fixed ideas. Hallucinations; thinks he is possessed of two persons or wills. Anxiety when walking, 
as if pursued. Profound melancholy and hypochondriasis, with tendency to use violent language. Brain-fag. Impaired memory. Absent mindedness. Very easily offended. Malicious; seems bent on wickedness. Lack of confidence in himself or others. Suspicious (Hyos). Clairaudient, hears voices far away or of the dead. Senile dementia. Absence of all moral restraint. Voluptuous itching; increased desire; seminal emissions without dreams. Prostatic discharge during stool.

c. Argentum nitricum: The patient is intellectual strong, patien complaint of memory loss, along with disturbed sense of reasoning. Patient is involved in foolish task, with strange conclusions. Patient experience strange illusions and hallucinations. His mind is full of useless and troublesome thoughts specially at night time, which makes him very anxious. Due to anxiety patients keeps on walking.Melancholia.Weak of memory, It seems time passes very slowly. Dulness of head, mental confusion ; dizziness ; tendency to fall sideways.

d. Caladium: Patient is unable to remember things, he is very forgetful with vague mind. Due to absentmindedness, he keep on searching different things. Concentration is very less due to which he is unable to put his ideas into speech. He has high desire for sex with relaxation of his organ. A state of total impotency. During intimacy there is urethral discharge. at times impotency is due to mental suppression. Severe itching on testcles.

e. Conium mac: Mental state is full of hysteria with the nervousness Patient complaint of weakness of muscles with trembling. Patient has very high desire for sex, but due to impotency he is not able to perform. Patient complaints of semen loss during sleep with or without dreams. Ejaculation is very painful- as if cutting with a knife due to acrid semen. Swelling and hardness.

f. Lycopodium: There is great desire to be alone. Despondent. Mentally and physically patient is very tired with complaint of chronic fatigue, with great aversion to his work. He is very forgetful, with dread of public appearance. Great Sensitivity, patient cries even when thanked. It is one of the most used medicines for impotency. Genital organ are feeble due to low vitality. Patient marries to live a normal life, but after marriage he finds he is sexually impotent without erections or very weak and short erections as if he is not a man. There is history of gonorrhoeal discharge with warts on male genitals. Patient is not trustworthy; he is very suspicious and find fault in every task. Patient is very timid with low self-confidence.

Classically selected tailored homoeopathy medicine helps in case of Male infertility. Well selected homeopathic medicine increase sperm count and also helps to overcome various complications related with male infertility.

There are more than 68 Homeopathic medicines which can be given to you based on your symptoms. Homeopathy treatment for Male infertility must be tailored for you so as to provide you deep pathological cure. Homeopathy treatment for male infertility is not only $100 \%$ safe but also very effective in large number of patients. The correct homeopathy medicines have to be selected by an experienced and a qualified Homeopathic doctor.

\section{Acknowledgments}

None.

\section{Conflicts of interest}

Author declares there are no conflicts of interest.

\section{Funding}

None. 